\title{
Association of Smoking and XPG, CYPIA1, OGG1, ERCC5, ERCC1, MMP2, and MMP9 Gene Polymorphisms with the early detection and occurrence of Laryngeal Squamous Carcinoma
}

\author{
Yi Zhu1, Luo Guo ${ }^{2}$, ShengZi Wang ${ }^{\circledR}$, Qun Yư ${ }^{3}$, JianXiong Lu ${ }^{3}$ \\ 1. Department of Radiation Oncology of Shanghai Eye and ENT Hospital of Fudan University, Shanghai, 200031, China \\ 2. Department of Experiment centre of Shanghai Eye and ENT Hospital of Fudan University, Shanghai,200031, China \\ 3. Department of TianPing health service centre of Shanghai,Shanghai,200031,China
}

$\square$ Corresponding author: ShengZi Wang, Mail address: Department of Radiation Oncology of Shanghai, Eye and ENT Hospital of Fudan University, Shanghai 200031, China. Telephone: 8618917761761; Email: shengziwang@fudan.edu.cn

(c) Ivyspring International Publisher. This is an open access article distributed under the terms of the Creative Commons Attribution (CC BY-NC) license (https:// creativecommons.org/licenses/by-nc/4.0/). See http://ivyspring.com/terms for full terms and conditions.

Received: 2017.09.16; Accepted: 2018.01.16; Published: 2018.02.28

\begin{abstract}
A total of 200 smoking-related laryngeal carcinoma patients with pathology confirmation from the Eye and ENT Hospital and 190 high-risk smokers were included in a survey. All of the participants had a smoking index greater than 400 (cigarettes/day*year.) We obtained data on clinical and baseline characteristics, and peripheral blood was obtained and subjected to DNA extraction to analyse the correlation between smoking and the occurrence of laryngeal carcinoma. We selected candidate genes and SNP fragments that were found to be closely associated with smoking-related tumours in preliminary studies. The selected candidate genes were XPG, CYPIA1, OGG1, ERCC5, ERCC1, MMP2, and MMP9. We then performed SNP sequencing using Sequenom SNP detection technology. Target genes and single nucleotide polymorphism (SNP) fragments were evaluated to analyse the correlation between genotype or allele and smoking-related laryngeal carcinoma and to identify susceptibility genes related to laryngeal carcinoma. The results included four main findings: (1) The smoking index differed significantly between laryngeal cancer patients and control subjects $(\mathrm{P}=0.0035)$. The risk of laryngeal cancer was increased among individuals with a smoking index greater than 600 cigarettes/day*year $(P=0.03)$. (2) The smoking index was significantly correlated with the T, N and clinical stages ( $P<0.05)$. (3) The polymorphisms CYPIA1-rs 1048943, rs4646421, and rs4646422 and MMP9-rs 17577 were significantly associated with laryngeal squamous carcinoma $(\mathrm{P}<0.05)$. (4) After stratifying the subjects by smoking degree, the GT genotype of ERCC1-rs2298881 was associated with a significantly greater risk of laryngeal carcinoma among heavy smokers $(P=0.04)$. The results suggest that smoking plays an important role in the occurrence and development of laryngeal squamous carcinoma; CYPIAI and MMP9 might be susceptibility gene SNPs for smoking-related laryngeal carcinoma, and ERCCl might play an important role in heavy smokers. The results of this study might help identify an early marker for the detection and prevention of laryngeal carcinoma.
\end{abstract}

Key words: laryngeal squamous carcinoma, polymorphism, susceptibility genes, SNP, smoking

\section{Introduction}

The incidence of laryngeal cancer is $1-5 \%$ of all malignancies and $65-70 \%$ of upper respiratory tract malignancies [1]. The larynx is an important organ, and individuals suffering from laryngeal cancer lose voice function. A previous epidemiological survey showed that the incidence of laryngeal cancer is 
influenced by several factors, including smoking, drinking, sex, age, eating habits, occupational factors, lifestyle and personality [2]. Smoking and excessive drinking have the largest effects on laryngeal cancer, with $80 \%$ of patients with laryngeal cancer having a history of smoking and alcohol use [3]. One study of laryngeal cancer found that the nitrosamines in tobacco lead to the up-regulation of DNA methylation, confirming that smoking is a risk factor for laryngeal cancer and demonstrating that the overexpression of DNA methylation in patients with laryngeal cancer is an important factor in the cancer's progression and metastasis [4]. Smoking is the most important factor in the study of laryngeal cancer. Tobacco and its transformants lead to oxidative damage of human DNA and causes DNA double-strand breaks. If this damage is not rapidly repaired, it promotes the occurrence of cancer. Many studies have shown that tobacco can reduce the expression of multiple DNA repair genes [5].

The occurrence of tumours is influenced by multiple factors and associations, both genes and environmental factors play an important role. However, the finding of familial clustering of specific tumours led to speculation regarding the existence of genetic characteristics affecting tumour occurrence that were independent of external factors. In addition, scientists observed that populations show variations in the occurrence of malignant tumours when exposed to the same risk factors. This finding suggested that individuals with different genetic backgrounds have different susceptibilities to malignancy under the same environmental exposure to risk factors. The term 'susceptibility' was thus introduced to describe an individual's risk of developing a disease due to genetic factors [6]. DNA sequence variations involving the change of a single nucleotide are known as single nucleotide polymorphisms (SNPs) and represent more than $90 \%$ of human genetic variation. Each SNP comprises 500-1000 base pairs, and SNPs have been used extensively to explore cancer and other genetic diseases [6]. Genotypic differences among individuals are known as polymorphisms and are widespread in populations. Genetic polymorphisms are third-generation genetic markers, and SNPs occur at more than 1\% frequency in a population [7]. At present, research on the relationships between gene polymorphisms and tumour occurrence is focused on the detection and analysis of several SNPs of target genes. Several studies have reported genes or SNP sites that occur at low frequencies and might be of interest for the early prevention and treatment of head and neck cancer.

The aim of the present study was to identify susceptibility genes by performing comparisons between laryngeal cancer patients with a history of smoking and high-risk control subjects with the same level of smoking. We conducted an epidemiological investigation of laryngeal cancer patients and normal smokers to understand the role of smoking in the development of laryngeal cancer and to study the susceptibility genes of individuals exposed to the same levels of environmental factors. The genetic susceptibility genes of tobacco-related head and neck cancer patients who were reported by previous scholars were compiled, and the high-risk genes and SNP fragments were evaluated to comprehensively and objectively investigate the genetic susceptibility of laryngeal cancer.

Table 1. Primer sequences (5'-3')

\begin{tabular}{llll}
\hline SNP_ID & 1st-PCRP & 2nd-PCRP & UEP_SEQ \\
\hline rs229881 & ACGTTGGATGATTCTATTGGCTCCGTCCCC & ACGTTGGATGGAGTCTGAGAGATGGACAAG & CCCCCGCCTTCCGTT \\
rs1052133 & ACGTTGGATGAGGTGCTGTTCAGTGCCGAC & ACGTTGGATGGAGTCTGGAACCCTTTCTGCG & CCGACCTGCGCCAAT \\
rs1047768 & ACGTTGGATGCAAGCACTTAAAGGAGTCCG & ACGTTGGATGAAGAGTTTGCAGAGCCGATG & AGTCCGGGATCGCCA \\
rs4646422 & ACGTTGGATGTCAGCATGTGCCCAATCAGA & ACGTTGGATGTGGGTAATCAGGGCCTCAAG & CCCAATCAGAGGCCAG \\
rs2304277 & ACGTTGGATGAAGGATCCCTAAGCAGTTAC & ACGTTGGATGCCATATCCCTAGTTCTGGTC & TCGTTACTGTGTGCCCA \\
rs3212964 & ACGTTGGATGATTTCACATCCTCTCTCCCG & ACGTTGGATGACAGCATGGGCAAAGGATGG & GGTCTCCCGTAGGGAGC \\
rs3787268 & ACGTTGGATGATCCTGGGCCATAGAGGATG & ACGTTGGATGCTTCCCAAACCACAGGACTT & GAGGATGTCGCTTAAAAC \\
rs3212961 & ACGTTGGATGCCTCTTGGAAGGGATTCAAC & ACGTTGGATGTCCAGGTGGATGTGGTAAGC & GGGAACAACCTCAAAGCCC \\
rs1048943 & ACGTTGGATGTGGGCAAGCGGAAGTGTATC & ACGTTGGATGCTGAATTCCACCCGTTGCAG & GAAGTGTATCGGTGAGACC \\
rs9928731 & ACGTTGGATGCCTTTCCTGGTCTGTATCTC & ACGTTGGATGGGAAAGCACGGAGCTCTTAA & TGTATCTCCACCTTTTAGTC \\
rs4771436 & ACGTTGGATGAATTTGTGCACTGCACTCCC & ACGTTGGATGAAGTTAGGCAGTGTACATTC & AATCCCTTTCACAGGGACTCTT \\
rs17577 & ACGTTGGATGTTGGACACGCACGACGTCTT & ACGTTGGATGTAGTGTGGTGTCTCACGAAG & GGGGACGACGTCTTCCAGTACC \\
rs1801320 & ACGTTGGATGAGCGAGTAGAGAAGTGGAGC & ACGTTGGATGAGCGCTCCTCTCTCCAGCA & GAGCGAAGTGGAGCGTAAGCCA \\
$\mathbf{r s 2 9 2 8 1 4 0}$ & ACGTTGGATGTCCAGCCTCATGACAGAGTG & ACGTTGGATGAGCTGCATTCTCCTCAGTCT & TCTCATACAAGGAACAAATAGAA \\
rs4646421 & ACGTTGGATGAGACTCCTTAGGGACACTTC & ACGTTGGATGCTTCATTGATCTGACCACTC & CGACATTTCCAGCTGCTGTCACAT \\
$\mathbf{r s 1 7 6 5 5}$ & ACGTTGGATGCTGAAGCACTGGTTTTTGGC & ACGTTGGATGCTTCATTGCTCAGAATCATC & GCAGTAAAGATGAACTTTCAGCAT \\
rs7201 & ACGTTGGATGGCAGGGCTGCGTTGAAAATA & ACGTTGGATGGATGCTGACTGTACTCCTC & GTGTCTGCGTTGAAAATATCAAAG \\
\hline
\end{tabular}




\section{Materials and Methods}

\section{Case and control groups}

Two hundred patients with laryngeal squamous carcinoma as determined via pathologic examination at the Eye and ENT Hospital between October 2014 and December 2016 were included in the study. Baseline and clinical data were collected, and patients with recurrence, distant metastasis or other serious diseases were excluded. One hundred ninety control subjects composed the tumour-free population. All of the patients and control subjects provided written, informed consent to participate in the study and had a long history of smoking. (According to WHO, when the smoking index more than 400cigarettes/day*year were identified as severe smokers. All of the participants in our study were severe smokers).

\section{Blood samples and genotyping}

Five millilitres of peripheral blood was collected from each patient and control and maintained at $-20^{\circ} \mathrm{C}$ until use. The phenol-chloroform extraction method was used to extract DNA from whole blood (QIAGEN DNA Mini and Blood Mini Kit). The genotypes and alleles of XPG (rs1047768), CYP1A1 (rs1048943/ rs4646421/rs4646422), OGG1 (rs1052133/rs2304277), ERCC5 (rsrs17655/rs4771436), RAD51 (rs1801320/ rs2928140), ERCC1 (rs2298881/rs3212961/rs3212964), MMP2 (rs7201/rs9928731), MMP9 (rs17577/rs3787 268) were analysed by polymerase chain reaction (PCR). The primer sequences are shown in Table 1 and were designed by using Sequenom ${ }^{\circledR}$ Assay Design version 3.1 software (ShengGong, Shanghai, China). The PCR procedure was as follows: initial denaturation at $95^{\circ} \mathrm{C}$ for $2 \mathrm{~min} ; 45$ cycles of amplification with denaturation at $95^{\circ} \mathrm{C}$ for $30 \mathrm{~s}$, annealing at $56^{\circ} \mathrm{C}$ for $30 \mathrm{~s}$, and extension at $72^{\circ} \mathrm{C}$ for 60 $\mathrm{s}$; followed by final extension at $72^{\circ} \mathrm{C}$ for $5 \mathrm{~min}$. Sequenom MassARRAY Assay Design 3.0 software was used to design a multiplexed SNP MassEXTEND assay. The genotypes and alleles were detected by using the Sequenom MassARRAY RS1000 platform following the recommended protocol, and the data were analysed by using Sequenom Typer 4.0 software.

\section{Statistical analysis}

Independent two-samples t-tests and CochranMantel-Haenszel tests were used to test for differences between the patients and controls in baseline characteristics. SPSS 17.0 software was used to analyse the general clinical data to assess the correlation between smoking and disease progression. HardyWeinberg equilibrium (HWE) was examined using a Chi-square $\left(X^{2}\right)$ test with one degree of freedom. The relationship between a genotype or allele and the occurrence of laryngeal carcinoma was analysed by using the SPSS 17.0 statistical software package. The genetic susceptibility genes associated with smokingrelated laryngeal carcinoma was then summarized according to the analysis results. Conditional multivariate logistic regression models were used to calculate odds ratios (ORs) and 95\% confidence intervals (CIs). The smoking index was used for stratification to analyse the influence of a genotype or allele on the risk of laryngeal carcinoma after controlling for the external factor of smoking. All tests were two-sided with a significance level of P-value $<0.05$.

\section{Results}

\section{Baseline and clinical characteristics of patient and control groups (Tables 2 and 3 )}

Table 2. Baseline characteristics of the patient and control groups

\begin{tabular}{lclll}
\hline Variable & Statistic & Patient $(\mathbf{n}=\mathbf{2 0 0})$ & Control $(\mathbf{n}=\mathbf{1 9 0})$ & P-value \\
\hline $\begin{array}{l}\text { Age group } \\
<65 \text { years }\end{array}$ & $\mathrm{n}(\%)$ & $114(57.00)$ & $123(64.74)$ & 0.1178 \\
$\begin{array}{l}>=65 \text { years } \\
\text { Gender }\end{array}$ & $\mathrm{n}(\%)$ & $86(43.00)$ & $67(35.26)$ & \\
$\begin{array}{l}\text { Male } \\
\text { Smoking status }\end{array}$ & $\mathrm{n}(\%)$ & $200(100.00)$ & $190(100.00)$ & $\mathrm{NA}$ \\
$\begin{array}{l}\text { Missing } \\
\text { Mild }\end{array}$ & $\mathrm{n}(\%)$ & & & $0.0035 \mathrm{c}$ \\
$\begin{array}{l}\text { Moderate } \\
\text { Severe }\end{array}$ & $\mathrm{n}(\%)$ & $71(35.50)$ & $94(49.47)$ & \\
\hline
\end{tabular}

Table 3. Baseline characteristics of the patient and control groups

\begin{tabular}{lcll}
\hline Variable & Statistic & Patient (n=200) & Control (n=190) \\
\hline T Stage & $\mathrm{n}(\%)$ & $12(6.00)$ & NA \\
1 & $\mathrm{n}(\%)$ & $59(29.50)$ & \\
2 & $\mathrm{n}(\%)$ & $68(34.00)$ & \\
3 & $\mathrm{n}(\%)$ & $61(30.50)$ & \\
4 & & NA \\
N Stage Lymph Nodes & & \\
0 & $\mathrm{n}(\%)$ & $67(33.50)$ & \\
1 & $\mathrm{n}(\%)$ & $60(30.00)$ & \\
2 & $\mathrm{n}(\%)$ & $72(36.00)$ & \\
3 & $\mathrm{n}(\%)$ & $1(0.50)$ & \\
Clinical Stage & & & \\
I & $\mathrm{n}(\%)$ & $12(6.00)$ & \\
II & $\mathrm{n}(\%)$ & $35(17.50)$ & \\
III & $\mathrm{n}(\%)$ & $65(32.50)$ & \\
IV & $\mathrm{n}(\%)$ & $88(44.00)$ & \\
\hline
\end{tabular}

\section{Associations of age and smoking level with laryngeal squamous carcinoma (Table 4)}

Table 4. Binary logistic regression of risk factors related to laryngeal cancer (full model)

\begin{tabular}{lllll}
\hline Factors & Odds Ratio & 95\% CI & P-value \\
\hline Intercept & & & & \\
Age Group & $>=65$ vs. $<65$ years & 1.260 & $0.822,1.929$ & 0.2886 \\
Smoking Index & $>=600$ vs. $<600 \mathrm{CY}$ & 1.816 & $1.060,3.113$ & 0.0300 \\
\hline
\end{tabular}

Note: $\mathrm{CY}=$ cigarettes $/$ day $^{*}$ years 
3. Significant relationships were observed for the T stage, $\mathbf{N}$ stage, and clinical stage with smoking level in laryngeal squamous carcinoma patients (Table 5 )

Table 5. Clinical characteristics and smoking level in patients

\begin{tabular}{|c|c|c|c|c|}
\hline & \multicolumn{3}{|c|}{ Smoking index category } & \multirow[b]{2}{*}{ P-value } \\
\hline & $\begin{array}{l}400=<,<800 \\
(N=71)\end{array}$ & $\begin{array}{l}800=<,<1200 \\
(N=80)\end{array}$ & $\begin{array}{l}>=1200 \\
(n=49)\end{array}$ & \\
\hline \multicolumn{4}{|l|}{ T Stage [n(\%)] } & $<0.0001 \mathrm{a}$ \\
\hline $\mathrm{T} 1$ & $9(12.7 \%)$ & $2(2.5 \%)$ & $1(2.0 \%)$ & \\
\hline $\mathrm{T} 2$ & $30(42.3 \%)$ & $21(26.3 \%)$ & $8(16.3 \%)$ & \\
\hline T3 & $18(25.4 \%)$ & $35(43.8 \%)$ & $15(30.6 \%)$ & \\
\hline $\mathrm{T} 4$ & $14(19.7 \%)$ & $22(27.5 \%)$ & $25(51.0 \%)$ & \\
\hline Total & 71 & 80 & 49 & \\
\hline \multicolumn{4}{|l|}{ N Stage $[n(\%)]$} & $<0.0001 \mathrm{a}$ \\
\hline No & $39(54.9 \%)$ & $17(21.3 \%)$ & $11(22.4 \%)$ & \\
\hline N1 & $18(25.4 \%)$ & $32(40.0 \%)$ & $10(20.4 \%)$ & \\
\hline N2 & $14(19.7 \%)$ & $30(37.5 \%)$ & $28(57.1 \%)$ & \\
\hline N3 & 0 & $1(1.3 \%)$ & 0 & \\
\hline Total & 71 & 80 & 49 & \\
\hline \multicolumn{3}{|c|}{ Clinical Stage $[\mathrm{n}(\%)]$} & NA & $<0.0001 \mathrm{a}$ \\
\hline I & $9(12.7 \%)$ & $2(2.5 \%)$ & $1(2.0 \%)$ & \\
\hline II & $26(36.6 \%)$ & $6(7.5 \%)$ & $3(6.1 \%)$ & \\
\hline III & $17(23.9 \%)$ & $35(43.8 \%)$ & $13(26.5 \%)$ & \\
\hline IV & $19(26.8 \%)$ & $37(46.3 \%)$ & $32(65.3 \%)$ & \\
\hline Total & 71 & 80 & 49 & \\
\hline
\end{tabular}

4. Hardy-Weinberg Test: None of the 17 genes deviated from Hardy-Weinberg equilibrium in the patient and control groups $(P>0.05)$ (Table 6)

Table 6. Genotype characteristics of selected SNPs

\begin{tabular}{llll}
\hline Gene & SNP & Alleles & HWE test (P-value) \\
\hline XPG & rs1047768 & $\mathrm{C} / \mathrm{T}$ & 0.463 \\
CYP1A1 & rs1048943 & $\mathrm{G} / \mathrm{A}$ & 0.406 \\
OGG1 & rs1052133 & $\mathrm{C} / \mathrm{G}$ & 0.794 \\
ERCC5 & rs17655 & $\mathrm{G} / \mathrm{C}$ & 0.962 \\
RAD51 & $\mathrm{rs} 1801320$ & $\mathrm{C} / \mathrm{G}$ & 0.951 \\
ERCC1 & $\mathrm{rs} 2298881$ & $\mathrm{G} / \mathrm{T}$ & 0.180 \\
ERCC1 & $\mathrm{rs} 3212961$ & $\mathrm{C} / \mathrm{A}$ & 0.251 \\
ERCC5 & $\mathrm{rs} 4771436$ & $\mathrm{G} / \mathrm{T}$ & 0.662 \\
MMP2 & $\mathrm{rs} 7201$ & $\mathrm{C} / \mathrm{A}$ & 0.659 \\
MMP2 & $\mathrm{rs} 9928731$ & $\mathrm{C} / \mathrm{T}$ & 0.688 \\
MMP9 & $\mathrm{rs} 17577$ & $\mathrm{~A} / \mathrm{G}$ & 0.150 \\
OGG1 & $\mathrm{rs} 2304277$ & $\mathrm{~A} / \mathrm{G}$ & 0.102 \\
RAD51 & $\mathrm{rs} 2928140$ & $\mathrm{G} / \mathrm{C}$ & 0.860 \\
ERCC1 & $\mathrm{rs} 3212964$ & $\mathrm{~T} / \mathrm{C}$ & 0.080 \\
MMP9 & $\mathrm{rs} 3787268$ & $\mathrm{~A} / \mathrm{G}$ & 0.656 \\
CYP1A1 & $\mathrm{rs} 4646421$ & $\mathrm{~A} / \mathrm{G}$ & 0.053 \\
CYP1A1 & $\mathrm{rs} 4646422$ & $\mathrm{C} / \mathrm{T}$ & 0.723 \\
\hline
\end{tabular}

5. The analysis of gene data showed that CYP1A1-rs1048943, CYP1A1-rs4646421, CYP1A1-rs4646422 and MMP9-rs17577 were closely related to the occurrence of laryngeal carcinoma and could thus be considered genetic susceptibility genes for the development and progression of laryngeal carcinoma (Table 7)

Table 7. Gene analysis results

\begin{tabular}{|c|c|c|c|c|c|}
\hline Gene & Test result & Patient (\%) & Control (\%) & OR(95\%CI)* & P-value \\
\hline \multirow[t]{5}{*}{ rs1047768 } & $\mathrm{CC}$ & $19(9.5)$ & $19(10.0)$ & 1(ref) & 0.98 \\
\hline & TT & $103(51.8)$ & 97 (51.1) & $1.06(0.53-2.12)$ & \\
\hline & $\mathrm{CT}$ & 77 (38.7) & 74 (38.9) & $1.04(0.51-2.12)$ & \\
\hline & C carrier & $115(28.9)$ & $112(29.5)$ & 1(ref) & 0.86 \\
\hline & $\mathrm{T}$ carrier & $283(71.1)$ & $268(70.5)$ & $1.03(0.76-1.41)$ & \\
\hline \multirow[t]{5}{*}{ rs1048943 } & CG & $13(6.6)$ & $9(4.7)$ & 1(ref) & 0.12 \\
\hline & AA & $110(55.6)$ & $125(65.8)$ & $0.61(0.25-1.48)$ & \\
\hline & GA & 75 (37.9) & $56(29.5)$ & $0.93(0.37-2.32)$ & \\
\hline & G carrier & $101(25.5)$ & 75 (19.5) & 1 (ref) & 0.04 \\
\hline & A carrier & $295(74.5)$ & $306(80.5)$ & $0.71(0.50-0.99)$ & \\
\hline \multirow[t]{5}{*}{ rs1052133 } & $\mathrm{CC}$ & $30(15.1)$ & 27 (14.2) & 1(ref) & 0.80 \\
\hline & GG & 74 (37.2) & 77 (40.5) & $0.86(0.47-1.59)$ & \\
\hline & CG & 95 (47.7) & $86(45.3)$ & $0.99(0.55-1.80)$ & \\
\hline & C carrier & 155 (37.6) & $140(35.5)$ & 1 (ref) & 0.55 \\
\hline & G carrier & $243(62.4)$ & $240(64.5)$ & $0.91(0.68-1.22)$ & \\
\hline \multirow[t]{5}{*}{ rs17577 } & AA & $4(2.0)$ & $4(2.1)$ & 1 (ref) & 0.06 \\
\hline & GG & $146(73.0)$ & 157 (82.6) & $0.93(0.23-3.79)$ & \\
\hline & AG & $50(25.0)$ & $29(15.3)$ & $1.72(0.40-7.42)$ & \\
\hline & A carrier & $58(14.5)$ & $37(9.7)$ & 1(ref) & 0.04 \\
\hline & G carrier & $342(85.5)$ & $343(90.3)$ & $0.63(0.41-0.99)$ & \\
\hline \multirow[t]{5}{*}{ rs17655 } & GG & $52(26.1)$ & $50(26.3)$ & 1 (ref) & 0.92 \\
\hline & $\mathrm{CC}$ & 45 (22.6) & $46(24.2)$ & $0.94(0.53-1.66)$ & \\
\hline & GC & $102(51.3)$ & $94(49.5)$ & $1.04(0.65-1.68)$ & \\
\hline & G carrier & $206(51.8)$ & $194(51.1)$ & 1 (ref) & 0.84 \\
\hline & C carrier & $192(48.2)$ & $186(48.9)$ & $0.97(0.73-1.29)$ & \\
\hline
\end{tabular}




\begin{tabular}{|c|c|c|c|c|c|}
\hline Gene & Test result & Patient (\%) & Control (\%) & OR $(95 \% \mathrm{CI})^{*}$ & P-value \\
\hline \multirow[t]{5}{*}{ rs1801320 } & $\mathrm{CC}$ & $2(1.0)$ & $5(2.6)$ & 1 (ref) & 0.36 \\
\hline & GG & $138(69.3)$ & $136(71.6)$ & $2.53(0.48-13.29)$ & \\
\hline & CG & $59(29.6)$ & $49(25.8)$ & $3.01(0.56-16.18)$ & \\
\hline & C carrier & $63(15.8)$ & $59(15.5)$ & $1(\mathrm{ref})$ & 0.91 \\
\hline & G carrier & $335(84.2)$ & $321(84.5)$ & $0.98(0.66-1.43)$ & \\
\hline \multirow[t]{5}{*}{ rs2928140 } & GG & $128(64.0)$ & $134(70.5)$ & 1 (ref) & 0.39 \\
\hline & $\mathrm{CC}$ & $5(2.5)$ & $4(2.1)$ & $1.31(0.34-4.98)$ & \\
\hline & GC & $67(33.5)$ & $52(27.4)$ & $1.35(0.87-2.09)$ & \\
\hline & G carrier & $323(80.8)$ & $320(84.2)$ & 1 (ref) & 0.20 \\
\hline & C carrier & $77(19.3)$ & $60(15.8)$ & $1.27(0.88-1.84)$ & \\
\hline \multirow[t]{5}{*}{ rs2304277 } & AA & $69(34.5)$ & $59(31.1)$ & 1 (ref) & 0.18 \\
\hline & GG & $39(19.5)$ & $27(14.2)$ & $1.24(0.68-2.25)$ & \\
\hline & AG & $92(46.0)$ & $104(54.7)$ & $0.76(0.48-1.18)$ & \\
\hline & A carrier & $230(57.5)$ & $222(58.4)$ & 1 (ref) & 0.79 \\
\hline & G carrier & $170(42.5)$ & 158 (41.6) & $1.04(0.78-1.39)$ & \\
\hline \multirow[t]{5}{*}{ rs2298881 } & GG & $56(28.1)$ & $68(35.8)$ & 1 (ref) & 0.27 \\
\hline & TT & $45(22.6)$ & $39(20.5)$ & $1.40(0.80-2.44)$ & \\
\hline & GT & $98(49.2)$ & $83(43.7)$ & $1.43(0.91-2.27)$ & \\
\hline & G carrier & $210(52.8)$ & $219(57.6)$ & 1 (ref) & 0.17 \\
\hline & $\mathrm{T}$ carrier & $188(47.2)$ & $161(42.4)$ & $1.22(0.92-1.61)$ & \\
\hline \multirow[t]{5}{*}{ rs3212961 } & $\mathrm{CC}$ & $46(23.2)$ & $58(30.5)$ & 1 (ref) & 0.27 \\
\hline & AA & $53(26.8)$ & $46(24.2)$ & $1.45(0.84-2.53)$ & \\
\hline & CA & $99(50.0)$ & $86(45.3)$ & $1.45(0.90-2.35)$ & \\
\hline & C carrier & $191(54.3)$ & $202(46.3)$ & 1 (ref) & 0.17 \\
\hline & A carrier & $205(45.7)$ & 178 (53.7) & $1.22(0.92-1.61)$ & \\
\hline \multirow[t]{5}{*}{ rs3212964 } & TT & $47(23.5)$ & $47(24.7)$ & 1 (ref) & 0.83 \\
\hline & $\mathrm{CC}$ & $68(34.0)$ & $68(35.8)$ & $1.00(0.59-1.69)$ & \\
\hline & TC & $85(42.5)$ & $75(39.5)$ & $1.13(0.68-1.89)$ & \\
\hline & $\mathrm{T}$ carrier & $179(44.8)$ & $169(44.5)$ & 1 (ref) & 0.94 \\
\hline & C carrier & $221(55.2)$ & 211 (55.5) & $0.99(0.75-1.31)$ & \\
\hline \multirow[t]{5}{*}{ rs3787268 } & $\mathrm{AA}$ & $32(16.0)$ & $24(12.6)$ & 1 (ref) & 0.58 \\
\hline & GG & $81(40.5)$ & $84(44.2)$ & $0.72(0.39-1.33)$ & \\
\hline & AG & $87(43.5)$ & $82(43.2)$ & $0.80(0.43-1.46)$ & \\
\hline & A carrier & $151(48.4)$ & $130(43.2)$ & 1 (ref) & 0.30 \\
\hline & G carrier & 249 (51.6) & $250(56.8)$ & $0.86(0.64-1.15)$ & \\
\hline \multirow[t]{5}{*}{ rs4771436 } & GG & $24(12.1)$ & $20(10.5)$ & 1 (ref) & 0.88 \\
\hline & TT & $93(46.7)$ & $92(48.4)$ & $0.84(0.44-1.63)$ & \\
\hline & GT & $82(41.2)$ & 78 (41.1) & $0.88(0.45-1.71)$ & \\
\hline & G carrier & $130(32.7)$ & $118(31.1)$ & 1 (ref) & 0.63 \\
\hline & $\mathrm{T}$ carrier & $268(67.3)$ & $262(68.9)$ & $0.93(0.68-1.25)$ & \\
\hline \multirow[t]{5}{*}{ rs4646421 } & AA & $45(22.5)$ & $21(11.1)$ & 1 (ref) & 0.01 \\
\hline & GG & $63(31.5)$ & $66(34.7)$ & $0.45(0.24-0.83)$ & \\
\hline & AG & $92(46.0)$ & $103(54.2)$ & $0.42(0.23-0.75)$ & \\
\hline & A carrier & $182(45.5)$ & $145(38.2)$ & 1 (ref) & 0.04 \\
\hline & G carrier & $218(54.5)$ & 235 (61.8) & $0.74(0.56-0.98)$ & \\
\hline \multirow[t]{5}{*}{ rs4646422 } & $\mathrm{CC}$ & 149 (74.5) & $122(64.2)$ & 1 (ref) & 0.02 \\
\hline & TT & $2(1.0)$ & $9(4.7)$ & $0.18(0.04-0.86)$ & \\
\hline & $\mathrm{CT}$ & $49(24.5)$ & $59(31.1)$ & $0.68(0.43-1.06)$ & \\
\hline & C carrier & $347(86.8)$ & 303 (79.7) & 1 (ref) & 0.009 \\
\hline & $\mathrm{T}$ carrier & $53(13.2)$ & $77(20.3)$ & $0.60(0.41-0.88)$ & \\
\hline \multirow[t]{5}{*}{ rs7201 } & $\mathrm{CC}$ & $12(6.0)$ & $7(3.7)$ & 1 (ref) & 0.50 \\
\hline & AA & $115(57.8)$ & 117 (61.6) & $0.57(0.22-1.51)$ & \\
\hline & CA & $72(36.2)$ & $66(34.7)$ & $0.64(0.24-1.71)$ & \\
\hline & C carrier & $96(24.1)$ & $80(21.1)$ & 1 (ref) & 0.31 \\
\hline & A carrier & 302 (75.9) & $300(78.9)$ & $0.84(0.60-1.18)$ & \\
\hline \multirow[t]{5}{*}{ rs9928731 } & $\mathrm{CC}$ & $43(21.6)$ & $46(24.3)$ & 1 (ref) & 0.53 \\
\hline & TT & $57(28.6)$ & $45(23.8)$ & $1.36(0.77-2.40)$ & \\
\hline & CT & $99(49.7)$ & $98(51.9)$ & $1.08(0.65-1.78)$ & \\
\hline & C carrier & $185(46.5)$ & $190(50.3)$ & 1 (ref) & 0.29 \\
\hline & $\mathrm{T}$ carrier & $213(53.5)$ & $188(49.7)$ & $1.16(0.88-1.54)$ & \\
\hline
\end{tabular}


5.1 The frequency of the rs 1048943 alleles significantly differed between the patient and control groups $(P=0.04)$. The results indicated that $A$ carriers had a 0.71 -fold higher risk of laryngeal carcinoma relative to G carriers (Table 8)

Table 8. Association between rs 1048943 and laryngeal carcinoma

\begin{tabular}{lllllll}
\hline Genotype and allele & \multicolumn{2}{l}{ Patient } & Control & $\mathrm{X}^{\mathbf{2}}$ & P-value & OR (95\%CI) \\
\hline Genotype GG & 13 & 9 & 4.28 & 0.12 & 1 \\
& AA & 110 & 125 & & & $0.61(0.25-1.48)$ \\
\multirow{6}{*}{ Allele } & GA & 75 & 56 & & & $0.93(0.37-2.32)$ \\
& G & 101 & 74 & 4.04 & 0.04 & 1 \\
& A & 295 & 306 & & & $0.71(0.50-0.99)$ \\
\hline
\end{tabular}

5.2 The frequency of the rs 17577 alleles significantly differed between the patient and control groups $(P=0.04)$. The results indicated that $G$ carriers had a 0.63 -fold higher risk of laryngeal carcinoma relative to A carriers (Table 9)

Table 9. Association between rs17577 and laryngeal carcinoma

\begin{tabular}{lllllll}
\hline Genotype and allele & Patient Control & $\mathbf{X}^{2}$ & P-value & OR (95\%CI) \\
\hline Genotype & AA & 4 & 4 & 5.73 & 0.06 & 1 \\
& GG & 146 & 157 & & & $0.93(0.23-3.79)$ \\
\multirow{6}{*}{ Allele } & AG & 50 & 29 & & & $1.72(0.40-7.42)$ \\
& A & 58 & 37 & 4.13 & 0.04 & 1 \\
& G & 342 & 343 & & & $0.63(0.41-0.99)$ \\
\hline
\end{tabular}

5.3 The frequencies of the genotypes and alleles of rs4646421 were significantly different between the patient and control groups $(\mathrm{P}<0.05)$. The $\mathrm{GG}$ genotype had a 0.45 -fold greater risk of laryngeal carcinoma relative to the $\mathrm{AA}$ genotype, whereas the AG genotype had a 0.42 -fold greater risk relative to the AA genotype $(P=0.01)$. In addition, $G$ carriers were associated with a 0.74 -fold higher risk of laryngeal carcinoma relative to $A$ carriers $(P=0.04)$ (Table 10)

Table 10. Association between rs4646421 and laryngeal carcinoma

\begin{tabular}{lllllll}
\hline Genotype and allele & \multicolumn{2}{l}{ Patient } & Control & $\chi^{2}$ & P-value & OR (95\%CI) \\
\hline Genotype & AA & 45 & 21 & 9.17 & 0.01 & 1 \\
& GG & 63 & 66 & & & $0.45(0.24-0.83)$ \\
\multirow{6}{*}{ Allele } & AG & 92 & 103 & & & $0.42(0.23-0.75)$ \\
& A & 182 & 145 & 4.31 & 0.04 & 1 \\
& G & 218 & 235 & & & $0.74(0.56-0.99)$ \\
\hline
\end{tabular}

5.4 The frequencies of the genotypes and alleles of rs4646422 were significantly different between the patient and control groups $(\mathrm{P}<0.05)$. Relative to the CC genotype, the TT genotype was associated with 0.18 -fold greater risk of laryngeal carcinoma, whereas the CT genotype was associated with a 0.68 -fold greater risk $(\mathrm{P}=0.02)$. T carriers had a 0.60 -fold greater risk of the disease relative to $C$ carriers $(P=0.009$ (Table 11))

Table 11. Association between rs4646422 and laryngeal carcinoma

\begin{tabular}{|c|c|c|c|c|c|c|}
\hline \multicolumn{2}{|c|}{ Genotype and allele } & \multirow{2}{*}{$\begin{array}{l}\text { Patient } \\
149\end{array}$} & \multirow{2}{*}{$\begin{array}{l}\text { Control } \\
122\end{array}$} & \multirow{2}{*}{$\begin{array}{l}x^{2} \\
9.17\end{array}$} & \multirow{2}{*}{$\begin{array}{l}\text { P-value } \\
0.02\end{array}$} & \multirow{2}{*}{$\begin{array}{l}\text { OR }(95 \% \mathrm{CI}) \\
1\end{array}$} \\
\hline Genotype & $\mathrm{CC}$ & & & & & \\
\hline & TT & 2 & 9 & & & $0.18(0.04-0.86)$ \\
\hline & CT & 49 & 59 & & & $0.68(0.43-1.06)$ \\
\hline \multirow[t]{2}{*}{ Allele } & C & 347 & 303 & 6.90 & 0.009 & 1 \\
\hline & $\mathrm{T}$ & 53 & 77 & & & $0.60(0.41-0.88)$ \\
\hline
\end{tabular}

6. We divided the patients and controls into three groups according to smoking level: mild (400-800 cigarettes/day*year), moderate (800-1200cigarettes/day*year) and severe smoking (>1200cigarettes/day*year). The analysis of genotype and smoking level showed that the GT genotype of rs2298881 was associated with a significantly higher risk of laryngeal carcinoma $(P=0.010)$. GT genotype carriers had an increased risk of laryngeal carcinoma as smoking level increased (Table 12)

Table 12. Analysis of gene data stratified by smoking level

\begin{tabular}{|c|c|c|c|c|c|c|c|}
\hline \multirow[b]{3}{*}{ Gene } & \multicolumn{7}{|c|}{ Smoking level } \\
\hline & & Mild & & Moderate & & Severe & \\
\hline & Genotype & OR (95\%CI) & P-value & OR $(95 \% \mathrm{CI})$ & $P$-value & OR (95\%CI) & P-value \\
\hline \multirow[t]{3}{*}{ rs1047768 } & $\mathrm{CC}$ & 1.00 & & 1.00 & & 1.00 & \\
\hline & TT & $0.87(0.28-2.70)$ & 0.988 & $1.10(0.35-3.45)$ & 0.996 & $0.40(0.04-4.11)$ & 0.385 \\
\hline & СТ & $0.75(0.23-2.41)$ & 0.588 & $1.22(0.38-3.92)$ & 0.699 & $0.54(0.05-5.90)$ & 0.829 \\
\hline \multirow[t]{3}{*}{ rs1048943 } & GG & 1.00 & & 1.00 & & 1.00 & \\
\hline & AA & $0.61(0.17-2.15)$ & 0.384 & $0.49(0.11-2.16)$ & 0.256 & $0.00(0.00-\mathrm{I})$ & 0.979 \\
\hline & GA & $0.74(0.20-2.76)$ & 0.916 & $0.67(0.14-3.16)$ & 0.920 & $0.00(0.00-\mathrm{I})$ & 0.983 \\
\hline rs1052133 & $\mathrm{CC}$ & 1.00 & & 1.00 & & 1.00 & \\
\hline
\end{tabular}




\begin{tabular}{|c|c|c|c|c|c|c|c|}
\hline \multirow[b]{3}{*}{ Gene } & \multicolumn{7}{|c|}{ Smoking level } \\
\hline & & Mild & & Moderate & & Severe & \\
\hline & Genotype & OR $(95 \% \mathrm{CI})$ & P-value & OR $(95 \% \mathrm{CI})$ & P-value & OR $(95 \% \mathrm{CI})$ & P-value \\
\hline \multirow{5}{*}{ rs17655 } & GG & $0.56(0.19-1.59)$ & 0.648 & $0.74(0.27-2.06)$ & 0.399 & $2.26(0.52-9.88)$ & 0.589 \\
\hline & CG & $0.44(0.15-1.24)$ & 0.151 & $1.04(0.39-2.76)$ & 0.595 & $2.77(0.65-11.81)$ & 0.274 \\
\hline & GG & 1.00 & & 1.00 & & 1.00 & \\
\hline & $\mathrm{CC}$ & $0.80(0.32-2.01)$ & 0.806 & $1.12(0.43-2.91)$ & 0.968 & $1.51(0.30-7.53)$ & 0.802 \\
\hline & GC & $0.77(0.34-1.76)$ & 0.673 & $1.29(0.60-2.75)$ & 0.559 & $1.62(0.44-5.90)$ & 0.602 \\
\hline \multirow[t]{3}{*}{ rs1801320 } & $\mathrm{CC}$ & 1.00 & & 1.00 & & 1.00 & \\
\hline & GG & $0.27(0.02-3.19)$ & 0.357 & $0.00(0.00-\mathrm{I})$ & 0.984 & $0.00(0.00-\mathrm{I})$ & 0.981 \\
\hline & CG & $0.25(0.02-3.09)$ & 0.298 & $0.00(0.00-\mathrm{I})$ & 0.983 & $0.00(0.00-\mathrm{I})$ & 0.979 \\
\hline \multirow[t]{3}{*}{ rs2298881 } & GG & 1.00 & & 1.00 & & 1.00 & \\
\hline & $\mathrm{TT}$ & $1.20(0.47-3.06)$ & 0.568 & $2.40(0.91-6.29)$ & 0.201 & $0.95(0.26-3.42)$ & 0.154 \\
\hline & GT & $0.89(0.41-1.92)$ & 0.543 & $1.90(0.88-4.09)$ & 0.554 & $5.55(1.41-21.81)$ & 0.010 \\
\hline \multirow[t]{3}{*}{ rs3212961 } & $\mathrm{CC}$ & 1.00 & & 1.00 & & 1.00 & \\
\hline & $\mathrm{AA}$ & $1.10(0.43-2.80)$ & 0.821 & $2.43(0.93-6.34)$ & 0.144 & $1.31(0.36-4.80)$ & 0.681 \\
\hline & $\mathrm{CA}$ & $1.01(0.44-2.34)$ & 0.923 & $1.73(0.78-3.85)$ & 0.761 & $2.81(0.83-9.51)$ & 0.109 \\
\hline \multirow[t]{3}{*}{ rs4771436 } & GG & 1.00 & & 1.00 & & 1.00 & \\
\hline & $\mathrm{TT}$ & $0.96(0.31-3.00)$ & 0.996 & $1.18(0.43-3.26)$ & 0.604 & $0.78(0.12-5.15)$ & 0.979 \\
\hline & GT & $0.91(0.28-2.93)$ & 0.861 & $0.96(0.34-2.71)$ & 0.743 & $0.59(0.09-3.77)$ & 0.511 \\
\hline \multirow[t]{3}{*}{ rs7201 } & $\mathrm{CC}$ & 1.00 & & 1.00 & & 1.00 & \\
\hline & $\mathrm{AA}$ & $0.92(0.20-4.31)$ & 0.881 & $0.23(0.02-2.16)$ & 0.254 & $1.36(0.18-10.13)$ & 0.723 \\
\hline & $\mathrm{CA}$ & $0.97(0.20-4.73)$ & 0.983 & $0.21(0.02-2.08)$ & 0.210 & $2.87(0.33-24.74)$ & 0.208 \\
\hline \multirow[t]{3}{*}{ rs9928731 } & $\mathrm{CC}$ & 1.00 & & 1.00 & & 1.00 & \\
\hline & $\mathrm{TT}$ & $0.88(0.34-2.23)$ & 0.679 & $1.83(0.64-5.20)$ & 0.154 & $2.41(0.55-10.50)$ & 0.443 \\
\hline & $\mathrm{CT}$ & $1.06(0.46-2.44)$ & 0.714 & $0.94(0.41-2.17)$ & 0.299 & $2.15(0.62-7.44)$ & 0.548 \\
\hline \multirow[t]{3}{*}{ rs17577 } & $\mathrm{AA}$ & 1.00 & & 1.00 & & 1.00 & \\
\hline & GG & $0.22(0.02-2.40)$ & 0.050 & $0.00(0.00-\mathrm{I})$ & 0.980 & $1.57(0.08-29.60)$ & 0.664 \\
\hline & AG & $0.66(0.06-7.66)$ & 0.634 & $0.00(0.00-\mathrm{I})$ & 0.979 & $1.20(0.05-27.20)$ & 0.962 \\
\hline \multirow[t]{3}{*}{ rs2304277 } & $\mathrm{AA}$ & 1.00 & & 1.00 & & 1.00 & \\
\hline & GG & $1.74(0.65-4.61)$ & 0.088 & $1.27(0.46-3.56)$ & 0.510 & $0.85(0.18-3.91)$ & 0.884 \\
\hline & AG & $0.67(0.31-1.43)$ & 0.053 & $0.87(0.41-1.84)$ & 0.465 & $0.58(0.19-1.79)$ & 0.397 \\
\hline \multirow[t]{3}{*}{ rs2928140 } & GG & 1.00 & & 1.00 & & 1.00 & \\
\hline & $\mathrm{CC}$ & $0.88(0.09-8.22)$ & 0.881 & $3.78(0.37-38.09)$ & 0.390 & & \\
\hline & GC & $1.09(0.53-2.22)$ & 0.816 & $1.88(0.89-3.97)$ & 0.960 & $1.67(0.48-5.86)$ & 0.420 \\
\hline \multirow[t]{3}{*}{ rs3212964 } & $\mathrm{TT}$ & 1.00 & & 1.00 & & 1.00 & \\
\hline & $\mathrm{CC}$ & $1.05(0.43-2.55)$ & 0.927 & $0.97(0.39-2.43)$ & 0.913 & $1.56(0.42-5.75)$ & 0.843 \\
\hline & $\mathrm{TC}$ & $1.18(0.52-2.71)$ & 0.677 & $1.02(0.41-2.54)$ & 0.915 & $1.95(0.54-7.03)$ & 0.407 \\
\hline \multirow[t]{3}{*}{ rs3787268 } & $\mathrm{AA}$ & 1.00 & & 1.00 & & 1.00 & \\
\hline & GG & $0.74(0.27-2.06)$ & 0.293 & $0.53(0.19-1.48)$ & 0.254 & $1.00(0.20-5.05)$ & 0.571 \\
\hline & AG & $1.21(0.44-3.29)$ & 0.355 & $0.64(0.23-1.81)$ & 0.743 & $0.53(0.10-2.66)$ & 0.262 \\
\hline \multirow[t]{3}{*}{ rs4646421 } & $\mathrm{AA}$ & 1.00 & & 1.00 & & 1.00 & \\
\hline & GG & $0.55(0.21-1.44)$ & 0.297 & $0.35(0.12-1.05)$ & 0.284 & $0.29(0.04-2.07)$ & 0.518 \\
\hline & AG & $0.66(0.27-1.65)$ & 0.744 & $0.29(0.10-0.81)$ & 0.047 & $0.21(0.04-1.19)$ & 0.103 \\
\hline \multirow[t]{3}{*}{ rs 4646422} & $\mathrm{CC}$ & 1.00 & & 1.00 & & 1.00 & \\
\hline & $\mathrm{TT}$ & $0.00(0.00-14 \mathrm{E} 84)$ & 0.918 & $0.31(0.03-3.16)$ & 0.431 & 3E4 (0.00-1E253) & 0.971 \\
\hline & CT & $0.78(0.38-1.61)$ & 0.921 & $0.63(0.29-1.36)$ & 0.864 & $0.68(0.19-2.49)$ & 0.970 \\
\hline
\end{tabular}

\section{Discussion}

According to the International Agency for Research on Cancer Globocan website (GLOBOCAN 2008), the standardized incidence of laryngeal cancer worldwide is $2.2 / 10$ million, and the standardized mortality rate is $1.2 / 10$ million. The incidence of laryngeal cancer is lower in China than in Europe and the United States; however, the disease remains a concern in China, with a standardized incidence of 1.4/10 million and a standardized mortality of $0.8 / 10$ million [8, 9]. Several studies have shown that smoking and excessive drinking have strong influences on laryngeal carcinoma. A previous survey showed that $80 \%$ of patients with laryngeal cancer had a history of smoking and alcohol abuse and that the risk of laryngeal cancer increases with increasing time and volume of these two factors [3]. The proportion of laryngeal cancer was increased significantly among those who smoked more than 20 cigarettes and drank more than $15 \mathrm{ml}$ of alcohol each day for at least 5 years [10]. Ramroth et al analysed 257 laryngeal cancer patients and 769 controls and showed that smoking was the most important factor influencing the occurrence of laryngeal cancer. Smokers have an increased incidence of laryngeal cancer, and non-smokers, including children, who are exposed to a smoking environment also have a higher incidence compared with those in a non-smoking environment [11]. However, although it is known that tobacco can promote the occurrence of laryngeal cancer, reports of the correlation between smoking 
level and laryngeal cancer are lacking. All of the participants in the present study had a history of long-term smoking. Our epidemiological results showed that the smoking level of patients with laryngeal carcinoma was significantly higher than that of individuals in the control group, who were without cancer. The regression analysis showed that the risk of laryngeal cancer was increased at a smoking level of more than 600 cigarettes/day*year, and the $\mathrm{T}$ stage, $\mathrm{N}$ stage and clinical stage of laryngeal carcinoma were significantly correlated with tobacco use. These results indicate that smoking not only promotes the occurrence of laryngeal carcinoma but also promotes its progression.

Earlier studies suggested that tumour development was mainly due to environmental factors. However, the finding of tumour aggregations in families raised the question of whether the occurrence of cancer was correlated with genetic factors. A researcher at Michigan University accidentally discovered a tumour family. The family comprised seven generations and total of 929 people as of 2002. The cancer incidence rate of this family was $12.4 \%$, which was higher than that of the general population, and most of the affected individuals had colorectal or endometrial cancer [12]. In addition to identifying such family aggregation phenomena, researchers also found that the influences of environmental factors varied among individuals such that exposure to the same risk factors did not invariably lead to cancer [6]. Therefore, we hypothesized that differences in genetic susceptibility contribute to variation in the occurrence of tumours. In our study, all of the subjects were exposed to the environmental factor of smoking to control for the influence of smoking. Our results suggested that CY1A1-rs1048943, CY1A1-rs4646421， CY1A1-s4646 422 and MMP9-rs17577 were closely related to susceptibility to laryngeal carcinoma. CYP1A1 is an enzyme encoded by the cytochrome gene and exerts aromatic hydrocarbon hydroxylase activity. CYP1A1 is one of the most important isoenzymes in the CYP450 enzyme system. Human CYP1A1 is located on chromosome 15, q22_q24, and it can catalyse PAH and other exogenous compounded active carcinogens. CYP1A1 is associated with nuclear DNA and causes mutations in cancer genes and tumour suppressor genes, promoting the occurrence of cancer [13]. To date, 133 candidate single nucleotide polymorphisms (SNPs) in human CYP1A1 have been found. Most studies of CYP1A1 gene polymorphisms and tumour susceptibility have focused on lung cancer and gastric cancer, with a smaller number of studies focused on oral cancer, laryngeal cancer and other head and neck cancer. In 2012, Sabitha et al found that a CYP1A1 gene mutation was closely related to cigarette smoking and that polymorphism of the CYP1A1 gene core region was closely related to the occurrence of tobacco-associated tumours. The CYP1A1 $\mathrm{m} 1 / \mathrm{m} 1$ and $\mathrm{w} 1 / \mathrm{m} 1$ genotypes were associated with an increased risk of head and neck cancer relative to the CYP1A1 $\mathrm{w} 1 / \mathrm{w} 1$ genotype. The CYP1A1 w2/m2 and $\mathrm{m} 2 / \mathrm{m} 2$ genotypes were also associated with a higher risk of head and neck cancer relative to the CYP1A1 w2/w2 genotype. The study authors suggested that CYP1A1 and its genotypes could serve as novel and important biomarkers for predicting and diagnosing head and neck cancers [14]. In addition, Lourenço et al studied 142 patients with head and neck squamous cell carcinoma and 142 control subjects and found that CYP1A1, GSTM1 and GSTT1 were closely related to head and neck cancer especially in patients with a severe smoking level [15]. A meta-analysis in 2016 found that carriers of the G allele for CYP1A1 rs1048943 and rs4646903 had a significantly increased risk of laryngeal cancer in Asian populations; 10 trials were included in the study, involving 748 patients with laryngeal cancer and 1558 control subjects [16]. These results are consistent with the present finding suggesting that allele G carriers at rs1048943 have an increased risk of laryngeal carcinoma. A recent study in Japan of CY1A1-rs1048943, rs4646421, rs4646422, GSTT1, and GSTM1 enrolled 457 patients with gastric cancer and 457 controls. The result showed that the rs4646422 polymorphism of CYP1A1 had a close association with gastric cancer in Japan and was associated with a significant increase in the risk of smoking, especially among individuals with more than 30 years of smoking [17]. These findings are consistent with the present result that CYP1A1-rs4646422 gene polymorphism was closely related to laryngeal carcinoma in the long-term smoking population.

MMPs degrade almost all extracellular matrix components and are closely related to tumour infiltration, invasion and metastasis. A few studies have focused on the correlation between laryngeal cancer and MMP expression level. In 2014, researchers analysed 48 tissues with laryngeal carcinoma and 15 tissues with non-cancerous laryngeal tissues for differences in CD147, MMP2 and MMP9 expression. In the laryngeal carcinoma tissues, the expression of CD147, MMP2 and MMP9 was significantly increased compared with that in the control group $(87.5 \%$, $75.0 \%$, and $79.2 \%$ vs. $26.7 \%, 6.7 \%$, and $33.3 \%$, respectively). The 5-year survival rate of laryngeal carcinoma was stratified according to the expression of CD147, MMP2 and MMP9. The 5-year survival rate was $25 \%$ in the high-expression group and $83.3 \%$ in the low-expression group. These results suggested 
that the expression of CD147, MMP2 and MMP9 was associated with the invasion, metastasis and prognosis of laryngeal carcinoma (16). In 2015, researchers reported that the expression levels of MMP2 and MMP9 were significantly higher in patients with laryngeal cancer than in normal, cancer-free patients. Their findings suggested that MMP2 expression was associated with lymph node metastasis, whereas MMP9 expression was significantly related to the $\mathrm{T}$ stage of laryngeal cancer [18]. To investigate the correlation of MMP9 expression in laryngeal squamous cell carcinoma tissues and prognosis, one study divided 196 patients into three groups according to MMP9 expression level. The authors of that study discussed the relationship between MMP9 expression and pathological grade, tumour stage and survival and suggested that MMP9 was an important biomarker of laryngeal squamous cell carcinoma and could predict prognosis [19]. In 2013, a study evaluated MMP9 gene polymorphism as a potential biomarker to predict breast cancer prognosis in Chinese women. The authors found that carriers of the rs3787268 GA + AA genotype and rs17577 GG genotype showed significantly reduced survival rates among breast cancer patients with ER (+) and Her-2 (-) [20]. Our results suggested that the $G$ allele of rs17577 had a protective effect compared with the A allele; this finding conflicts with the findings of the breast cancer study. These conflicting results might arise from study differences in the tumour site and pathological type, and this topic warrants further research. To date, little research has been conducted on the relationship between MMP9 gene polymorphisms and head and neck tumours. Most related studies have focused on the expression level of MMP9 in tumour issues. Therefore, our results are relevant for the early prediction of laryngeal cancer occurrence.

Few studies have investigated the relationship between ERCC1 polymorphisms and head and neck tumours; most studies have focused on colorectal, gastric, and lung cancers [21, 22]. A 2015 study using RegulomeDB technology found that ERCC1 had a total of 364 SNP sites. The authors of that study investigated four priority sites, rs298881, rs1049739, rs10415949, and rs6509214, which have been found to be associated with lung cancer prognosis. They found that rs2298881 C>A expression suggested poor survival and OS-free survival (DFS) for non-small cell lung cancer patients who had accepted surgery [23]. This result is consistent with our finding that the GT genotype at ERCC1-rs2298881 was associated with an increased risk of laryngeal cancer in heavy smokers after stratified analysis. A study of colorectal cancer that analysed the sites ERCC1-rs2298881, rs11615, and rs2276466 concluded that carriers of the C allele of ERCC1-rs2298881 had an increased risk of colorectal cancer and that this allele might be an important biomarker for colorectal cancer [24]. A meta-analysis reported the correlations of ERCC1-rs3212986 and ERCC1-rs11615 with head and neck tumours in 2015. This study included 910 head and neck cancer patients and 1337 control subjects from seven trials. The authors of that study concluded that the ERCC1-rs3212986 polymorphism in Asian populations was a predictor of head and neck cancer, whereas the ERCC1-rs11615 polymorphism was a risk factor for head and neck tumours, particularly laryngeal tumours [25]. One study investigated eight nucleotide polymorphisms of six genes in the context of smoking-related laryngeal squamous carcinoma. The researchers showed that the interactions between the ERCC1-rs11615 and ERCC5-rs17655 polymorphisms and smoking could promote the development of laryngeal cancer and that the ERCC1-rs1615 polymorphism was significantly associated with drinking habits [26]. In 2014, 12 single nucleotide polymorphisms (SNPs) of the NER gene were reported to be closely related to laryngeal cancer development. The results suggested that the CC genotype or C allele carriers of ERCC1-rs11615 had a significantly higher risk of tumours relative to the TT genotype, especially in the population with smoking history. The risk of laryngeal cancer was also higher among GG genotype or G allele carriers of ERCC5rs17655 compared with CC genotype carriers [27]. The rs17655 polymorphism was also included in our analyses, but no significant relationships were identified; further research on this polymorphism is warranted.

The results of our study suggest that smoking is an important factor in the development and progression of laryngeal cancer. The CYP1A1rs1048943, CYP1A1-rs4646421, CYP1A1-rs4646422 and MMP9-rs17577 polymorphisms are closely related to the susceptibility of laryngeal squamous carcinoma. In addition, the results indicate that the ERCC1-rs2298881 polymorphism has clinical monitoring value among individuals with a severe smoking level.

\section{Abbreviations}

SNP: single nucleotide polymorphism; NER: nucleotide excision repair.

\section{Acknowledgements}

Thanks for support of the research project of prevention and control of laryngeal cancer (SHDC 12012317). 


\section{Competing Interests}

The authors have declared that no competing interest exists.

\section{References}

1. Yu H, Xin Y-F, Duan X-D, et al. Dynamic analysis of epidemiology and aetiology on laryngeal carcinoma. Progr Mod Biomed. 2007; 7: 393-5.

2. Wang C, Li Q, Wang Y, et al. Case-control study on risk factors of laryngeal cancer in Heilongiiang Province. Lin Chung Er Bi Yan Hou Tou Jing Wai Ke za Zhi. 2011; 25: 1117-9.

3. Pantel M, Guntinas-Lichius O. [Laryngeal carcinoma: epidemiology, risk factors and survival]. HNO. 2012; 60: 32-40.

4. Wang J, Xu Y, Li J, et al. The tobacco-specific carcinogen NNK induces DNA methyltransferase 1 accumulation in laryngeal carcinoma. Oral Oncol. 2012; 48: 541-6.

5. Romanowicz-Makowska H, Smolarz B, Gajęcka M, et al. Polymorphism of the DNA repair genes RAD51 and XRCC2 in smoking- and drinking-related laryngeal cancer in a Polish population. Arch Med Sci. 2012; 8: 1065-75.

6. Hemminki K, Försti A, Lorenzo Bermejo J. Etiologic impact of known cancer susceptibility genes. Mutat Res. 2008; 658: 42-54.

7. Hou RZ, Zhao JS. Association of single nucleotide polymorphism of ERCC1 and XPG with colourectal cancer risk and interactin with tobacco use. JiLin: The University of JiLin; 2015.

8. Ling-bin D,Weimin M,Wanqin C, et al. Incidence and mortality of larynx cancer in China during 2003-2007. Chin J Epidemiol 2012; 33: 395-8.

9. WHO. GLOBOCAN. Cancer Incidence and Mortality Worldwide In 2008. USA: International Agency for Research on Cancer. 2008.

10. Guoxiang L, Li L, Haiyan C. Analysis of laryngeal cancer aetiology. Clin Med. 2011; 31: 98.

11. Ramroth H, Dietz A, Becher H. Environmental tobacco smoke and laryngeal cancer: results from a population-based case-control study. Eur Arch Otorhinolaryngol. 2008; 265: 1367-71.

12. Douglas JA, Gruber SB, Meister KA, et al. History and molecular genetics of Lynch syndrome in family G: a century later. JAMA. 2005; 294: 2195-202.

13. Nelson DR, Koymans L, Kamataki T, et al. P450 superfamily: update on new sequences, gene mapping, accession numbers and nomenclature. Pharmacogenetics. 1996; 6: 1-42.

14. Sabitha K, Reddy MV, Jamil K. Smoking related risk involved in individuals carrying genetic variants of CYP1A1 gene in head and neck cancer. Cancer Epidemiol. 2010; 34: 587-92.

15. Lourenço GJ, Silva EF, Rinck-Junior JA, et al. CYP1A1, GSTM1 and GSTT1 polymorphisms, tobacco and alcohol status and risk of head and neck squamous cell carcinoma. Tumor Biol. 2011; 32: 1209-15.

16. Zeng W, Li Y, Lu E, et al. CYP1A1 RS1048943 and RS4646903 polymorphisms associated with laryngeal cancer susceptibility among Asian populations: a meta-analysis. J Cell Mol Med. 2016; 20: 287-93.

17. Hidaka A, Sasazuki S, Matsuo K, et al. CYP1A1, GSTM1 and GSTT1 genetic polymorphisms and gastric cancer risk among Japanese: a nested case-control study within a large-scale population-based prospective study. Int J Cancer. 2016; 139: 759-68.

18. Lotfi A, Mohammadi G, Saniee L, et al. Serum level of matrix metalloproteinase- 2 and -9 in patients with laryngeal squamous cell carcinoma and clinical significance. Asian Pac J Cancer Prev. 2015; 16: 6749-51.

19. Colović Z, Pesutić-Pisac V, Poljak NK, et al. Expression of matrix metalloproteinase- 9 in patients with squamous cell carcinoma of the larynx. Coll Antropol. 2013; 37: 151-5.

20. Fu F, Wang C, Chen LM, et al. The influence of functional polymorphisms in matrix metalloproteinase 9 on survival of breast cancer patients in a Chinese population. DNA Cell Biol. 2013; 32: 274-82.

21. Ye W, Kumar R, Bacova G, et al. The XPD 751Gln allele is associated with an increased risk for esophageal adenocarcinoma: a population-based case-control study in Sweden. Carcinogenesis. 2006; 27: 1835-41.

22. Zhou RM, Li Y, Wang N, et al. Correlation of XPC Ala499 Val and Lys939Gln polymorphisms to risk of oesophageal squamous cell carcinoma and gastric cardiac adenocarcinoma. Ai Zheng. 2006; 25: 1113-9.

23. Lee SY, Hong MJ, Jeon HS, et al. Functional intronic ERCC1 polymorphism from regulomeDB can predict survival in lung cancer after surgery. Oncotarget. 2015; 6: 24522-32.

24. Yang H, Li G, Li WF. Association between ERCC1 and XPF polymorphisms and risk of colorectal cancer. Genet Mol Res. 2015; 14: 700-5.

25. Ding YW, Gao X, Ye DX, et al. Association of ERCC1 polymorphisms (RS3212986 and RS11615) with the risk of head and neck carcinomas based on case-control studies. Clin Transl Oncol. 2015; 17: 710-9.

26. $\mathrm{Lu} \mathrm{B}, \mathrm{Li} \mathrm{J}, \mathrm{Gao} \mathrm{Q}$, et al. Laryngeal cancer risk and common single nucleotide polymorphisms in nucleotide excision repair pathway genes ERCC1, ERCC2, ERCC3, ERCC4, ERCC5 and XPA. Gene. 2014; 542: 64-8.

27. $\mathrm{Li} \mathrm{X}, \mathrm{Xu} \mathrm{J}$, Yang $\mathrm{X}$, et al. Association of single nucleotide polymorphisms of nucleotide excision repair genes with laryngeal cancer risk and interaction with cigarette smoking and alcohol drinking. Tumor Biol. 2014; 35: 4659-65. 